\title{
Macro-coherent radiative emission of neutrino pair between parity-even atomic states
}

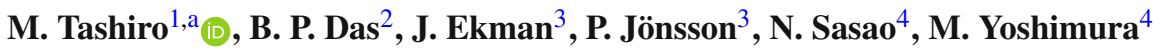 \\ ${ }^{1}$ Department of Applied Chemistry, Toyo University, Kujirai 2100, Kawagoe, Saitama 350-8585, Japan \\ ${ }^{2}$ Department of Physics, Tokyo Institute of Technology, 2-12-1-H86 Ookayama, Meguro-ku, Tokyo 152-8550, Japan \\ ${ }^{3}$ Department of Materials Science and Applied Mathematics, Malmö University, 20506 Malmö, Sweden \\ ${ }^{4}$ Research Institute for Interdisciplinary Science, Okayama University, 700-8530 Okayama, Japan
}

Received: 6 June 2019 / Accepted: 25 October 2019 / Published online: 10 November 2019

(C) The Author(s) 2019

\begin{abstract}
A new scheme to determine the neutrino mass matrix is proposed using atomic de-excitation between two states of a few eV energy spacing. The determination of the smallest neutrino mass of the order of $1 \mathrm{meV}$ and neutrino mass type, Majorana or Dirac, becomes possible, if one can coherently excite more than 1 gram of atoms using two lasers.
\end{abstract}

\section{Introduction}

Despite of remarkable success in oscillation experiments [1], neutrino physics has left more conundrums than what it has discovered and already established: major conundrums are how small the lightest neutrino mass is and whether neutrinos are Majorana or Dirac type of fermions (the Majorana/Dirac distinction). These two items are linked to important issues in macro- and micro- worlds: how our matter dominated universe was created (the problem of baryon asymmetry of the present universe) and the unified theory beyond the standard $S U(3) \times S U(2) \times U(1)$ gauge theory. In this respect it is crucially important to invent new experimental schemes of neutrino physics beyond the established technology based on nuclear targets, since they release too large energy of a few to several $\mathrm{MeV}$, much larger than expected small neutrino masses in the sub-eV range.

One possible scheme is to use a target system of available energy closer to the expected range of neutrino masses $\left(0.1 \sim 10^{-3}\right) \mathrm{eV}$. It was recently suggested by a few of the authors that isolated atomic systems might be a solution provided that an effective mechanism of coherent amplification of event rates is realized [2]. Translated to the single atomic

a e-mail: tashiro046@toyo.jp rate, this scheme enhances the rate by the total number of phase-coherent atoms. One of the problems in the proposed scheme is however scarce candidate atoms: the best candidate in terms of rate so far found is Xe gas, but due to a large required laser energy (in sum $\sim 8 \mathrm{eV}$ ) the Xe scheme may encounter a great challenge of copious ionization loss with present laser technology.

A related important question is how many target atoms are prepared: high-density atomic target in solids is an obvious choice, but various relaxation processes arising in solids may destroy coherence. A possible solution is to encapsulate atoms, which may maintain isolated features of atoms in vacuum, yet may not possess relaxation phenomena present in ordinary solids. We propose here a new scheme to evade this difficulty.

We use the natural unit of $\hbar=c=1$ throughout the present paper unless otherwise stated.

\section{Radiative emission of neutrino pair: use of new interaction pieces}

We consider electroweak process of atomic de-excitation from a metastable excited state $|e\rangle$ to the ground state $|g\rangle$, $|e\rangle \rightarrow|g\rangle+\gamma+\nu \bar{v}$, with $\gamma$ a photon and $\nu \bar{v}$ a neutrino-pair. This process is described by the second order perturbation theory of combined electric dipole (E1) photon emission and four-Fermi type of weak process of neutrino pair emission. The coherently amplified process of this type has been termed RENP (Radiative Emission of Neutrino Pair) [2]. The phasecoherence over a macroscopic body of atoms has been experimentally verified in second order QED process to achieve a rate enhancement of the order of $10^{18}$ in agreement with theoretical expectation [3-5]. With macro-coherence of phases, the probability amplitudes obey 


$$
\begin{aligned}
& \sum_{a} e^{i\left(\mathbf{p}_{e g}-\mathbf{k}_{\gamma}-\mathbf{p}_{1}-\mathbf{p}_{2}\right) \cdot \mathbf{x}_{a}} \mathcal{A}\left(\mathbf{x}_{a}\right) \\
& \simeq \frac{N}{V}(2 \pi)^{3} \delta^{3}\left(\mathbf{p}_{e g}-\mathbf{k}_{\gamma}-\mathbf{p}_{1}-\mathbf{p}_{2}\right) \mathcal{A}_{0},
\end{aligned}
$$

where $\mathbf{p}_{e g}, \mathbf{k}_{\gamma}$ are wave vectors imprinted at laser excitation and emitted photon, and $\mathbf{p}_{i}(i=1,2)$ are momenta of the emitted neutrino-pair. Emergence of a macroscopic quantity, the atomic number density $n=N / V$, is ensured provided phases of atomic wave functions $\mathcal{A}\left(\mathbf{x}_{a}\right)=\mathcal{A}_{0}$ are common and not random over sites.

Since we use in the present work new pieces of electronneutrino interaction, we shall recapitulate results of the established electroweak theory of three flavors extended to accommodate finite neutrino masses. Adding neutral current and charged current interactions after Fierz transformation, the neutrino pair emission is described by the four-Fermi type interaction hamiltonian density,

$$
\begin{aligned}
& \frac{G_{F}}{\sqrt{2}} \Sigma_{i j} \Sigma_{\alpha} \bar{v}_{i} \gamma_{\alpha}\left(1-\gamma_{5}\right) \nu_{j} \bar{e}\left(\gamma^{\alpha} c_{i j}-\gamma^{\alpha} \gamma_{5} b_{i j}\right) e, \\
& c_{i j}=U_{e i}^{*} U_{e j}-\frac{1}{2}\left(1-4 \sin ^{2} \theta_{w}\right) \delta_{i j}, \\
& b_{i j}=U_{e i}^{*} U_{e j}-\frac{1}{2} \delta_{i j},
\end{aligned}
$$

$v_{i}(i=1,2,3)$ denoting neutrino mass eigenstates of masses $m_{i}$. The $3 \times 3$ matrix element $U_{a i}(a=e, \mu, \tau)$ describes neutrino mass mixing. Experimentally, $1-4 \sin ^{2} \theta_{W} \sim$ $0.0460 \pm 0.0064$.

There may be two RENP possibilities depending on which electron operators are used: (1) spatial component of axial vector current in Eq. (2), (2) time component of axial vector or spatial component of vector current. The temporal part of vector current does not contribute much due to orthogonality of wave functions, which is justified when electrons move in atoms non-relativistically. The dominant atomic electron contribution arises from the spatial part of axial vector given by the spin operator, $e^{\dagger} \alpha \gamma_{5} e=e^{\dagger} \Sigma e(\Sigma$ being the $4 \times 4$ Pauli matrix). This is dominant since there are no mixing between large and small components of Dirac 4-spinor wave functions. Combined with electric dipole (E1) photon emission, the angular momentum and parity change of atomic electrons is of the type, $\Delta J^{P}=0^{-}, 1^{-}, 2^{-}$, and hence may be called parity-odd RENP. The best initial atomic state in terms of rates is $\mathrm{Xe}\left(J^{P}=2^{-}\right)$. There is however a problem due to a large excitation energy $\sim 8.3 \mathrm{eV}$, such as a close elusive ionization level.

On the other hand, there are many atomic candidates for parity-even transitions between states having excitation energies much less than in the parity-odd transition case. For this purpose we shall study parity-even RENP which have smaller rates, but may have better chances to be realized in simpler experiments. The sub-dominant contributions arise from either of two ways of taking electron operators: the spatial part of vector, $\bar{e} \boldsymbol{\gamma} e=e^{\dagger} \boldsymbol{\alpha} e$, and zero-th component of axial vector, $e^{\dagger} \gamma_{5} e$. Both of these are suppressed by the velocity of atomic electrons, of the order of the fine structure constant $\sim 1 / 137$.

\section{Atomic target and photon energy spectrum for parity-even RENP}

We shall consider as a candidate atom of parity-even RENP, $\mathrm{Au}$ neutral atom or similar $\mathrm{Cu}$. Its level structure near the ground state is depicted in Fig. 1. According to [6] Au may well be isolated in encapsulated fullerene from environments and maintain essential features of $\mathrm{Au}$ in vacuum.

In the RENP process of $|e\rangle \rightarrow|g\rangle+\gamma+v_{i} \bar{v}_{j}$, neutrinopair $v_{i} \bar{v}_{j}$ of mass eigenstate is extremely difficult to detect, hence we rely on the photon energy spectrum to extract properties of massive neutrinos. In actual RENP experiments we excite atoms in the ground state by irradiating two counterpropagating lasers of frequencies close to half the level spacing, $\epsilon_{e g} / 2$, which have very good energy resolutions much less than $O(1 \mathrm{meV})$. Along with a trigger laser we watch whether RENP occurs or not. What is crucial for determination of energy resolution in experiments is the laser frequency resolution rather than resolution of detected photon energy. This way one can decompose amplitudes of neutrino pair emission into neutrino energy eigenstates instead of flavor eigenstates $v_{e}$. With finite neutrino masses the energy and the momentum conservation of coherent RENP gives rise to six thresholds $\omega_{i j}, i, j=1,2,3$, which are shifted from the massless neutrino value to $\omega \leq \omega_{i j}, \omega_{i j}=$ $\epsilon_{e g} / 2-\left(m_{i}+m_{j}\right)^{2} /\left(2 \epsilon_{e g}\right)$ [2]. Obviously, $\epsilon_{e g}>2 m_{3}$ must be obeyed to detect a neutrino-pair of largest mass $m_{3}$. Below,we will discuss in some detail how these threshold locations $\omega_{i j}$ are manipulated by excitation lasers.

We consider Au excited state of $|e\rangle=5 d^{9} 6 s^{2}$, decaying to the ground state $|g\rangle=5 d^{10} 6 s$. Intermediate states of $|p\rangle=$ $5 d^{10} n p, 5 d^{9} 6 s n p(n=6,7,8, \ldots)$ and $|p\rangle=n p^{5} 5 d^{10} 6 s^{2}$ of a hole state $(n=3,4,5)$ appear in the second order perturbation theory where $n$ is the principal quantum number. Energy levels of these states are at $\epsilon_{e g}=\epsilon\left(5 d^{9} 6 s^{2}{ }^{2} D_{3 / 2}\right)=$ $2.658 \mathrm{eV}$ and $\epsilon\left(5 d^{10} 6 p^{2} P_{3 / 2}\right)=5.105 \mathrm{eV}$.

RENP amplitudes in the perturbation theory are for $\gamma_{5}$ parity-even RENP,

$$
\begin{aligned}
& \frac{G_{F}}{\sqrt{2}} b_{i j} \mathcal{N}_{i j} \mathcal{M}(\omega) \equiv \frac{G_{F}}{\sqrt{2}} b_{i j} \mathcal{N}_{i j} \\
& \quad \times \sum_{p}\left(\frac{\langle g|\mathbf{d} \cdot \mathbf{E}| p\rangle\left\langle p\left|\gamma_{5}\right| e\right\rangle}{E_{g}-E_{p}+\omega}+\frac{\left\langle g\left|\gamma_{5}\right| p\right\rangle\langle p|\mathbf{d} \cdot \mathbf{E}| e\rangle}{E_{e}-E_{p}-\omega}\right),
\end{aligned}
$$




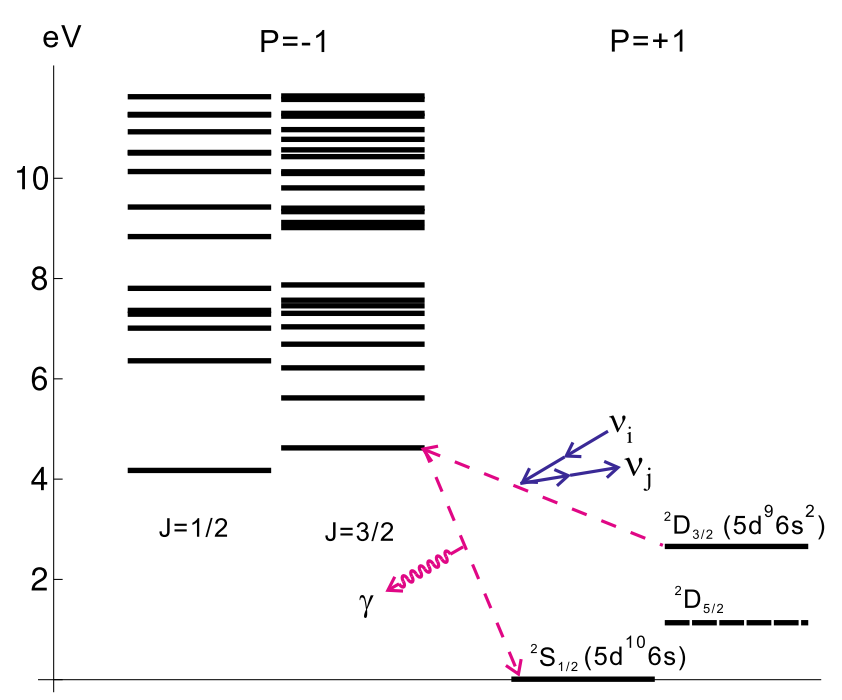

Fig. 1 Representative Au energy levels of different parities $P= \pm 1$ for RENP for ${ }^{2} D_{3 / 2} \rightarrow{ }^{2} S_{1 / 2}+\gamma+v \bar{\nu}$. The transition occurs via various $P=-1$ intermediate states; among those, $15 J=1 / 2$ and $25 J=3 / 2$ states are taken into the actual calculation. The dashed lines show an example of considered transitions, in which the transition amplitude is a product of a neutrino pair emission from $|e\rangle={ }^{2} D_{3 / 2}$ to $|p\rangle={ }^{2} P_{3 / 2}$ and an E1 radiative decay from $|p\rangle$ to $|g\rangle={ }^{2} S_{1 / 2}$

and for $\boldsymbol{\alpha}$ parity-even RENP,

$$
\begin{aligned}
& \frac{G_{F}}{\sqrt{2}} c_{i j} \mathcal{N}_{i j} \cdot \mathcal{M}(\omega) \equiv \frac{G_{F}}{\sqrt{2}} c_{i j} \mathcal{N}_{i j} . \\
& \sum_{p}\left(\frac{\langle g|\mathbf{d} \cdot \mathbf{E}| p\rangle\langle p|\boldsymbol{\alpha}| e\rangle}{E_{g}-E_{p}+\omega}+\frac{\langle g|\boldsymbol{\alpha}| p\rangle\langle p|\mathbf{d} \cdot \mathbf{E}| e\rangle}{E_{e}-E_{p}-\omega}\right) .
\end{aligned}
$$

Atomic parts of amplitudes, $\mathcal{M}(\omega)$ and $\mathcal{M}(\omega)$, are factored out of neutrino-pair emission parts, $\mathcal{N}_{i j}, \mathcal{N}_{i j}$. Neutrino-pair current $\left(\mathcal{N}_{i j}, \mathcal{N}_{i j}\right)=\bar{v}_{i} \gamma_{\alpha}\left(1-\gamma_{5}\right) v_{j}$ forms a 4-vector where $v_{j}, \bar{v}_{i}$ are neutrino plane wave functions of definite helicities and momenta. The two amplitudes given here are in principle comparable in magnitude, since both $\gamma_{5}$ and $\boldsymbol{\alpha}$ matrices have small and large component mixtures of 2 -spinors. Two types of parity-even RENP amplitudes never interfere when neutrino momenta are integrated, and one can discuss two rates arising from $\gamma_{5}$ and $\boldsymbol{\alpha}$ separately.

The idea we use to manipulate effectively threshold locations is to exploit the phase memory at laser excitation of atoms. With good quality of lasers of wave vectors, $\mathbf{k}_{1}, \mathbf{k}_{2}$, excited atoms maintain imprinted phases of $e^{i\left(\mathbf{k}_{1}+\mathbf{k}_{2}\right) \cdot \mathbf{x}}$. This may be regarded as an initial atom having a momentum, $\mathbf{k}_{1}+\mathbf{k}_{2}=\mathbf{p}_{e g}$, hence with macro-coherence the momentum conservation is changed to $\mathbf{p}_{e g}-\mathbf{k}-\mathbf{p}_{1}-\mathbf{p}_{2}=0$ instead of $\mathbf{k}+\mathbf{p}_{1}+\mathbf{p}_{2}=0$ where $\mathbf{p}_{j}, j=1,2$ momenta of neutrino pair and $\mathbf{k}$ the momentum of observed photon. This further changes the effective mass of the neutrino pair to $\sqrt{s}$ with $s=q^{2}$ and $q \equiv\left(q_{0}, \mathbf{q}\right)=\left(\epsilon_{e g}-\omega, \mathbf{p}_{e g}-\mathbf{k}\right)$, which may be smaller than the value without the imprinted phase, if $\left|\mathbf{p}_{e g}-\mathbf{k}\right|>|\mathbf{k}|$. We term this case boosted RENP [7].
Spectrum rate formula of boosted RENP for $\gamma_{5}$ parity-even case is given by $\Gamma=\sum_{i j} \Gamma_{i j}$, with

$$
\begin{aligned}
\Gamma_{i j}= & \frac{2 G_{F}^{2}}{\pi}|\mathcal{M}(\omega)|^{2} n^{2} V \\
& \times \int_{E_{-}}^{E_{+}} d E_{1} J_{i j}\left(E_{1}, \epsilon_{e g}-\omega-E_{1}\right) \theta\left(\sqrt{s}-m_{i}-m_{j}\right),
\end{aligned}
$$

$$
\begin{aligned}
J_{i j}\left(E_{1}, E_{2}\right)= & \frac{1}{|\mathbf{q}|}\left[\left|b_{i j}\right|^{2}\left(2 E_{1} E_{2}-\frac{s-m_{i}^{2}-m_{j}^{2}}{2}\right)\right. \\
& \left.+\delta_{M}\left(\Re b_{i j}^{2}\right) m_{i} m_{j}\right],
\end{aligned}
$$

where $E_{ \pm}$is the maximum and the minimum neutrino energy, and $\delta_{M}=1\left(\delta_{M}=0\right)$ is applied for the Majorana (Dirac) neutrino. The trigger laser field $\mathbf{E}$ in $\mathcal{M}(\omega)$ in Eq. (7) should be understood as $\rho_{e g} \mathbf{E}$ where $\rho_{e g}$ is coherence developed in target atoms. It is convenient to introduce a dimension-less parameter $\eta$ defined as $\left|\left\langle\rho_{e g} \mathbf{E}\right\rangle\right|^{2}=\omega n \eta / 2$ [7]. The factor $\eta$ is coherence averaged over target atoms, typically of order $10^{-3} \sim 10^{-6}$ from computations of [8]. In numerical computations below we use parallel or anti-parallel configuration in which the initial wave vector $\mathbf{p}_{e g}$ is taken along $\mathbf{k}$, with its magnitude by $p_{e g}=r \epsilon_{e g},-1 \leq r \leq 1$. For $\mathbf{p}_{e g}$ parallel to $\mathbf{k}$ the neutrino-pair production thresholds are at $\omega_{i j}=\frac{1+r}{2} \epsilon_{e g}-\frac{\left(m_{i}+m_{j}\right)^{2}}{2(1-r) \epsilon_{e g}}$. All six thresholds can be observed if the condition $r^{2}<1-4\left(m_{1} / \epsilon_{e g}\right)^{2}$ with $m_{1}$ is the smallest neutrino mass is satisfied.

The bench mark rate unit of parity-even RENP is estimated as a product of squared atomic matrix elements of order $10^{-3}$ in the atomic unit, the neutrino-pair integral of order $O(1 \sim$ $10^{-2}$ ) $\mathrm{eV}^{2}$ from Fig. 2 , and $G_{F}^{2} / 2 \pi$, which gives $\sim 10^{-6} \mathrm{~s}^{-1}$, taking $n=10^{22} \mathrm{~cm}^{-3}, V=10^{2} \mathrm{~cm}^{3}$ and $\eta=1$.

\section{Details of atomic calculation}

We now turn to atomic parts of amplitude $\mathcal{M}(\omega), \mathcal{M}(\omega)$. Eqs. (5) and (6) were evaluated for neutral gold, where we selected the $5 d^{9} 6 s^{2}\left({ }^{2} D_{3 / 2}\right)$ and $5 d^{10} 6 s\left({ }^{2} S_{1 / 2}\right)$ as the $|e\rangle$ and $|g\rangle$ states, respectively. For the $|p\rangle$ states in Eqs. (5) and (6), fifteen odd-parity $J=1 / 2$ valence excited states and twentyfour odd-parity $\mathrm{J}=3 / 2$ states were considered. In addition, $n p^{5}(n=3,4,5)$ odd-parity $\mathrm{J}=1 / 2$ and $3 / 2$ core-excited states were also included in the calculation. The $2 p^{5}$ coreexcited states were not included because of the program restriction, but its effect on the results are expected to be small since the excitation energies are much higher than the other valence or core-excited states.

The wave functions for these atomic states were calculated based on the multi-configuration Dirac-HartreeFock method and the relativistic configuration interaction 
method [9] implemented in the GRASP2K package [10], [11]. The wave functions, i.e., the atomic state functions, for these atomic states were represented by linear combinations of configuration state functions, which were constructed from single-particle Dirac orbitals. The single-particle Dirac orbitals were determined by the multi-configuration DiracHartree-Fock method, whereas the expansion coefficients of the linear combination were calculated by the relativistic configuration interaction method. In the first step of the calculation, using the multi-configuration Dirac-HartreeFock method, the $|g\rangle$ state was represented by the linear combination of the $5 d^{10} 6 s$ and $5 d^{9} 6 p^{2}$ configurations, and $|e\rangle$ by the $5 d^{9} 6 s^{2}$ and $5 d^{9} 6 p^{2}$ configurations, while $|p\rangle$ by the $5 d^{9} 6 s 6 p, 5 d^{10} 6 p$ and $5 d^{9} 6 p 6 d$ configurations. The inner orbitals were treated as inactive occupied core-orbitals. These configurations will be referred as multi-reference set hereafter. Then the self-consistent field procedure was applied to optimize both the expansion coefficients for the multi-reference configurations and the Dirac orbitals simultaneously. To correct for dynamic electron correlation effects, separate calculations for the $|g\rangle,|e\rangle$, and $|p\rangle$ states were performed with expansions including CSFs obtained by single (S) and double (D) excitations from the multi-reference set to active sets of orbitals up to $8 s, 10 p, 8 d, 6 f, 6 g$ and $6 h$. The core-valence and core-core correlations involving the $5 d^{10}$ core were included in addition to the valence correlations. In the last step, relativistic configuration interaction calculations were performed in which the Breit interaction [9], vacuum polarization [12], and self-energy [12] were considered. The transition matrix elements of the electric dipole, $\gamma_{5}$, and $\boldsymbol{\alpha}$ operators in Eqs. (5) and (6) were calculated by the reduced matrix elements, evaluated by the bi-orthogonalized atomic state functions, using the Wigner-Eckart theorem [11].

Results show that the zero-th component of axial vector contribution in the neutrino-pair emission vertex is much larger (by $\sim O(10)$ at $\omega \leq 3 \mathrm{eV}$ ) than the spatial component of vector contribution. We shall use the fitting function [13] arising $\gamma_{5}$ vertex alone for simplicity in the following.

\section{Results of RENP photon spectrum}

We present numerical results in Figs. 2 and 3 in the presence of an initial phase memory $r$. For these calculations of spectrum shapes we take squared neutrino mixing factors $\left|U_{e i}\right|^{2}(\mathrm{i}=1,2,3)$, hence $\left|b_{i j}\right|^{2}$, calculated from neutrino oscillation data [1]. CP violating (CPV) phases, $\delta$ for Dirac neutrino and $\delta, \alpha, \beta$ for Majorana neutrino, appear in $U_{e i} U_{e j}^{*}(i \neq j)$, and they are experimentally unknown. Other parameters determined by neutrino oscillation experiments are two mass squared differences, $\delta m_{i j}^{2}$. We assume in our analysis a particular smallest neutrino mass, and calculate other masses from these differences. Spectrum results are

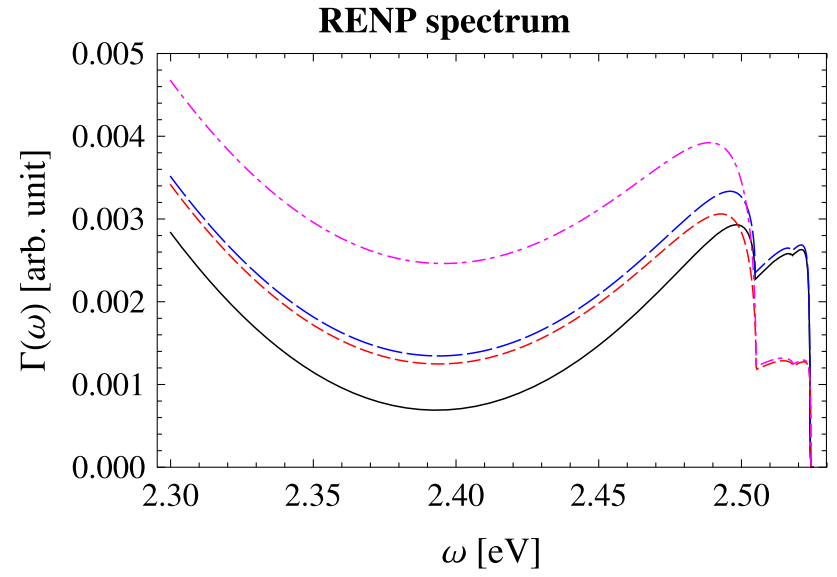

Fig. 2 Comparison of Dirac and Majorana spectra for the inverted (IO) and normal ordering (NO) cases with a parallel initial phase memory of $r=0.9$ : NO Dirac rate in solid black, NO Majorana in long-dashed blue, IO Dirac in short-dashed red, and IO Majorana in dash-dotted magenta for the smallest mass of $10 \mathrm{meV}$ and assuming vanishing $\mathrm{CP}$ violating phases. Absolute rate value is obtained by multiplying $10^{-6} \mathrm{~s}^{-1}$ for $n=10^{2} \mathrm{~cm}^{-3}, V=10^{2} \mathrm{~cm}^{3}, \eta=1$

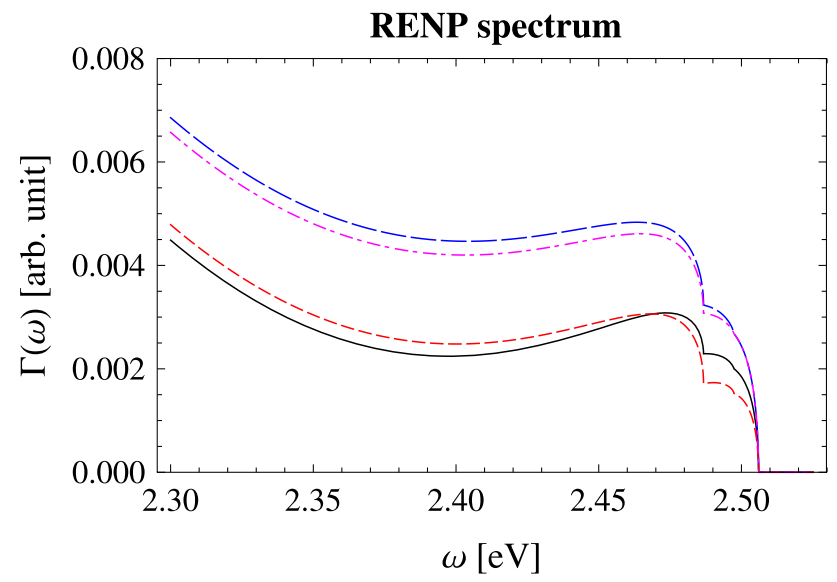

Fig. $3 \mathrm{CPV}$ phase $(\alpha, \beta-\delta)$ dependence of spectrum: Comparison of normal ordering (NO) spectra with a parallel initial phase memory of $r=0.9$ : Dirac in solid black, Majorana $(\alpha=0, \beta-\delta=0)$ in long-dashed blue, Majorana $(\alpha=\pi / 2, \beta-\delta=0)$ in short-dashed red, and Majorana ( $\alpha=0, \beta-\delta=\pi / 2$ ) in dash-dotted magenta for the smallest mass of $50 \mathrm{meV}$

shown both for normal ordering (NO) neutrino mass pattern and inverted ordering (IO) [1], which is experimentally undetermined so far.

A few comments we would like to make are

(1) the smallest neutrino mass determination is easiest at $\omega_{12}$ threshold in which the weight factor $\left|b_{12}\right|^{2}=0.405$ is largest,

(2) the Majorana/Dirac distinction is easiest after $\omega_{33}$ threshold opens with $\left|b_{33}\right|^{2}=0.227$, 
(3) unlike parity-odd RENP, parity-even RENP in the present work gives larger Majorana pair emission rates than Dirac pair emission,

(4) all other thresholds have much smaller (by $O(1 / 10)$ ) weights, but they are in principle detectable with finer resolutions of high statistics data, since two thresholds, $\omega_{12}, \omega_{33}$, making up most of the summed weights, $\sum_{i j}\left|b_{i j}\right|^{2}=3 / 4$,

(5) The term $\Re b_{i j}^{2}$ in Eq. (8) gives CPV phase dependences on $\alpha$ and $\beta-\delta$. It is largest for $\alpha$, since $b_{12} \propto e^{-i \alpha}$ and the phase independence of $b_{33}$. Dependence on $\beta-\delta$ is less sensitive due to their appearance in $U_{\rho i} U_{\rho j}^{*}(i \neq j)$ of flavors $\rho=\mu, \tau$.

The most serious background arises for macro-coherence involving QED processes, and for parity-even RENP discussed in the present work the background is macro-coherent four-photon process called McQ4 [14]. How to suppress this background is discussed in [15].

Unambiguous detection of atomic neutrino far above six thresholds is expected to give an overall neutrino mass scale, although the smallest neutrino mass measurement needs more dedicated efforts. In this sense the discovery of neutrino-pair production in atomic de-excitation is surely a breakthrough in neutrino physics.

In summary, we proposed a new experimental method of measuring the smallest neutrino mass and determining whether neutrinos are of Majorana or Dirac type, and presented results of how to extract neutrino properties by measuring emitted photon energy spectrum. Isolated neutral atoms in solid environment is necessary to prepare large enough targets in actual experiments.

Acknowledgements This research was partially supported by Grantin-Aid 16K05307(MT) and 16H00939(MT), 15H02093 (NS), 17H028 95 (MY), from the Ministry of Education, Culture, Sports, Science, and Technology of Japan. JE and PJ have been supported by the Swedish Research Council (VR) under contract 2015-04842. JE's visit to Tokyo Institute of Technology to initiate collaboration on the work reported here was supported by a grant from MEXT Japan under the auspices of The Program for Promoting the Enhancement of Research Universities.
Data Availability Statement This manuscript has no associated data or the data will not be deposited. [Authors' comment: The present article is not a experimental study and no experimental data has been listed.]

Open Access This article is distributed under the terms of the Creative Commons Attribution 4.0 International License (http://creativecomm ons.org/licenses/by/4.0/), which permits unrestricted use, distribution, and reproduction in any medium, provided you give appropriate credit to the original author(s) and the source, provide a link to the Creative Commons license, and indicate if changes were made.

Funded by SCOAP ${ }^{3}$.

\section{References}

1. Particle Data Group Collaboration, M. Tanabashi et al., Phys. Rev. D 98, 030001 (2018)

2. A. Fukumi et al., Prog. Theor. Exp. Phys. (2012) 04D002, and earlier references cited therein

3. Y. Miyamoto et al., PTEP, 113C01 (2014)

4. Y. Miyamoto et al., PTEP, $081 \mathrm{C01}$ (2015)

5. T. Hiraki et al., arXiv:1806.04005 [physics.atom-ph], and J. Phys. B 52, 045401 (2019)

6. S. Saito, Y. Sugaya, and M. Toyoda. Private communication

7. M. Tanaka, K. Tsumura, N. Sasao, M. Yoshimura, Phys. Rev. D 96, 113005 (2017)

8. M. Yoshimura, N. Sasao, M. Tanaka, Phys. Rev. A 86, 013812 (2012)

9. I.P. Grant, Relativistic quantum theory of atoms and molecules (Springer, New York, 2007)

10. P. Jönsson, X. He, C. Froese Fischer, I.P. Grant, Comput. Phys. Commun. 177, 597 (2007)

11. P. Jönsson, G. Gaigalas, J. Bieron, C. Froese Fischer, I.P. Grant, Comput. Phys. Commun. 184, 2197 (2013)

12. P.J. Mohr, G. Plunien, G. Soff, Phys. Rept. 293, 227 (1998)

13. The fitting formula of amplitude $|\mathcal{M}(\omega)|^{2}$ used in spectrum computations is $a_{0}+a_{1}(x-0.05)+a_{2}(x-0.05)^{2}+a_{3}(x-0.05)^{3}+$ $a_{4}(x-0.05)^{4}$, with $a_{0}=0.00103776, a_{1}=-0.00700889$, $a_{2}=0.136222, a_{3}=-1.08185, a_{4}=0.846076$ in terms of the atomic unit of $x \simeq \omega / 27.2 \mathrm{eV}$

14. M. Yoshimura, N. Sasao, M. Tanaka, PTEP, 053 B06 (2015)

15. M. Tanaka, K. Tsumura, N. Sasao, M. Yoshimura, PTEP, $043 B 03$ (2017) 